\title{
Embryonal Neuromesodermal Progenitors for Caudal Central Nervous System and Tissue Development
}

\author{
Mohammed R. Shaker, Ju-Hyun Lee, ${ }^{2}$ Woong Sun ${ }^{2}$ \\ Australian Institute for Bioengineering and Nanotechnology, The University of Queensland, Queensland, Australia \\ Department of Anatomy, ${ }^{2}$ Brain Korea 21 Plus Program for Biomedical Science, Korea University College of Medicine, Seoul, Korea
}

Neuromesodermal progenitors (NMPs) constitute a bipotent cell population that generates a wide variety of trunk cell and tissue types during embryonic development. Derivatives of NMPs include both mesodermal lineage cells such as muscles and vertebral bones, and neural lineage cells such as neural crests and central nervous system neurons. Such diverse lineage potential combined with a limited capacity for self-renewal, which persists during axial elongation, demonstrates that NMPs are a major source of trunk tissues. This review describes the identification and characterization of NMPs across multiple species. We also discuss key cellular and molecular steps for generating neural and mesodermal cells for building up the elongating trunk tissue.

Key Words : Neuromesodermal progenitors · Axial elongation · Spinal cord development · Neurulation · Neural tube.

\section{INTRODUCTION}

The precursors of the central nervous system (CNS) that give rise to the brain and spinal cord are developmentally derived from the neural tube ${ }^{40}$. The neural tube is formed during an embryonic process called neurulation, which consists of two phases : primary neurulation that executes neural plate folding to form the brain and most of the spinal neural tube (up to the lumbar level) (Fig. 1A and C), and secondary neurulation, which contributes to neural tube elongation to meet the demand of the longitudinal elongation of embryos (Fig. 1B and $\mathrm{C}$ ). The fate of the three germ layers is fixed during early gastrulation stages, for example the ectodermal layer generates the CNS and neural crest-derived tissues, and the mesodermal layer gives rise to the notochord, skeleton, and trunk muscles. However, clonal analysis revealed different embryonic lineages that contribute to the anteroposterior neural tube axis $^{50)}$. In particular, neuroectodermal cells are the major population of the anterior neural tube corresponding to the brain and upper spinal cord (Fig. 1C), and undergo primary neurulation (Fig. 1A). On the other hand, the caudal spinal neural tube is primarily derived from neuromesodermal progenitors (NMPs) (Fig. 1C) $)^{44)}$.

These NMPs progressively add new neural and mesodermal lineage cells to build the posterior tissues (CNS, skeleton, and muscles) of the body after gastrulation. In support of this notion, the posterior neural tube can form in the absence of an anterior neural tube ${ }^{6}$. We have recently illustrated the poten-

- Received : December 23, 2020 •Revised : January 8, 2021 •Accepted : January 28, 2021

- Address for reprints : Woong Sun

Department of Anatomy, Brain Korea 21 Plus Program for Biomedical Science, Korea University College of Medicine, 73 Inchon-ro, Seongbuk-gu, Seoul 02841, Korea Tel : +82-2-2286-1404, Fax : +82-2-2286-1387, E-mail : woongsun@korea.ac.kr, ORCID : https://orcid.org/0000-0003-1792-4894 
Primary neurulation
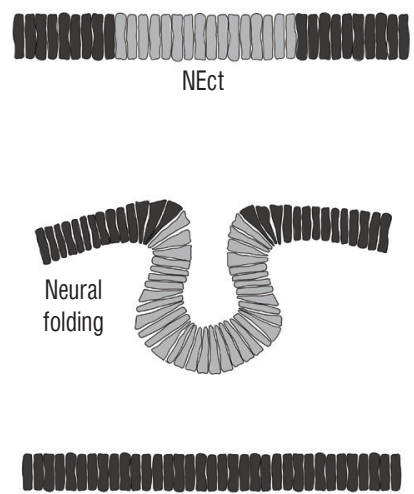

(A)

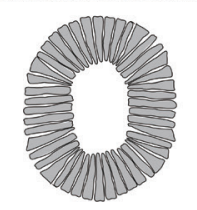

Primary neural tube
Secondary neurulation
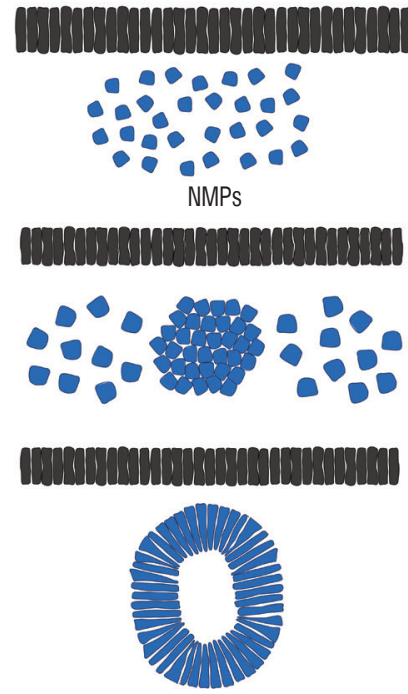

Secondary neural tube

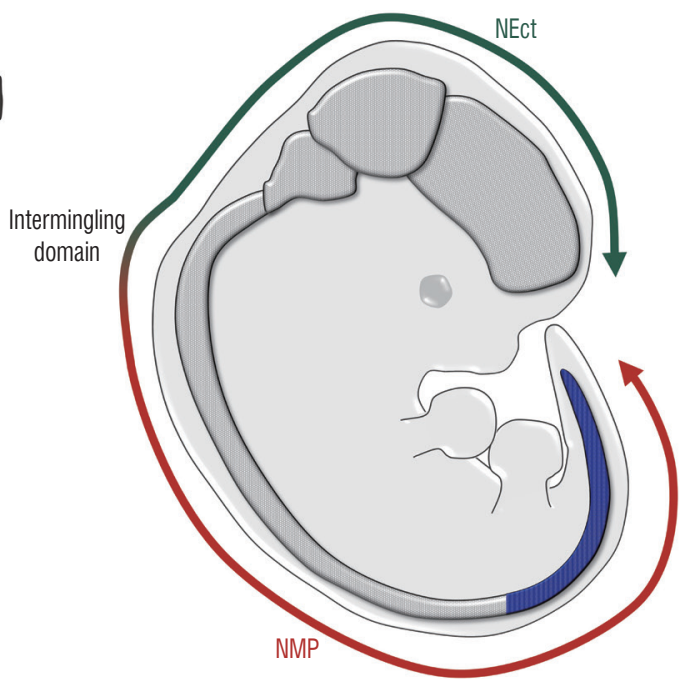

Primary neurulation

Secondary neurulation

Fig. 1. The process and lineage of neurulation in vertebrate embryos. A and B: Comparison of primary and secondary neurulation. $A$ : Transition in the morphology of neuroepithelium derived from the neuroectodermal cells during primary neurulation to establish the primary neural tube. The neuroepithelium initially undergoes neural folding that bends to adhere and fuse at both ends forming the primary neural tube. Mouse primary neurulation occurs between E8-E10 ${ }^{47}$. Chick primary neurulation occurs between $\mathrm{HH} 4-\mathrm{HH} 14$, whereas human primary neurulation takes place between Carnegie stages 8-12 $2^{37}$. B : Organization of cells at the onset (up) and completion (below) of secondary neurulation in vertebrates. The tail bud cells which are NMPs, differentiate into pre-neural cells that aggregate and integrate with the primary neural tube during axial elongation. Mouse secondary neurulation starts at E12 and ends at E14 ${ }^{47}$, whereas in chick and humans, it ends at $\mathrm{HH} 35^{26)}$ and Carnegie stage $18^{377}$, respectively. Although the term secondary neural tube refers to the product of secondary neurulation, secondary neurulation occurs during axial elongation and it contributes to the elongation of the neural tube. Therefore, secondary neurulation is conceptually equal to the axial elongation. $\mathrm{C}$ : The central nervous system development model of neuroectodermal and neuromesodermal lineage contribution toward body neural axis formation and extension in mouse. The neuroectodermal cells contribute to the brain and brachial development. The mouse NMPs generate the tail in addition to the spinal cord. NEct : neuroectodermal cells, NMPs : neuromesodermal progenitors, $\mathrm{HH}$ : Hamburger and Hamilton.

tial of NMPs to generate the trunk tissue up to the brachial level $^{44)}$. It is likely that neural cells derived from NMPs intermingle with neural cells of neuroectodermal origin at the brachial spinal cord level (Fig. 1C). However, co-lineage tracing of neuroectodermal cells as well as NMPs would first identify the level of CNS with junctional lineages, and second, illustrate whether there is a preference for neuroectodermal cells and NMPs toward subsequent neural progenies. Therefore, the nervous systems of the head and trunk are derived from distinct developmental origins. This recent identification of NMPs in vertebrates suggests that germ layer specification is not restricted to primary gastrulation, but continues up to the somitogenesis stages. The process of neural tube elongation is different with respect to morphogenesis and the end-product of developed tissues across species. Chick and human NMPs generate pre-neural cells that undergo cavitation to form a neural tube that eventually adheres to the primary neural tube ${ }^{46,52)}$, which develops the spinal cord up to the lumbar level. The mouse pre-neural cells derived from NMPs appear to behave differently; they first polarize laterally and migrate toward the primary neural tube and integrate for elongation to proceed. This process gives rise to tail formation in mice ${ }^{43}$. The recent identification of NMPs has fascinated developmental biologists, who apply multi-disciplinary approaches to investigate further. In this short review, we summarize the identification, lineage differentiation, and molecular niche of NMPs in the caudal part of the embryo.

\section{NMPS AND VERTEBRATE AXIAL ELONGATION}

During development, NMPs arise from the caudal lateral epiblast and adjacent node-streak border within the primary streak. As development proceeds, they are detected in the 
chordoneural hinge of the tail bud and continuously contribute to the neural and mesodermal tissues of the elongating tail $^{1)}$. Therefore, NMPs are generally defined as a bipotent cell population that is capable of producing neural and mesodermal fate tissues. Comparative analysis suggests that the spatiotemporal distribution and function of NMPs differ across species. In amniote mouse and chick models, the NMPs expand during trunk development and gradually get depleted until the termination of the elongating tail ${ }^{4,44)}$. This aligns with an in vitro study showing mouse neural progenitors derived from depleting NMPs at E12 to have a higher tendency for spontaneous neuronal differentiation with low self-renewal potency ${ }^{43)}$. However, Xenopus and zebrafish amniote NMPs exhibit progressive depletion after primary neurulation.

Experiments using lineage tracing and fluorescent labeling with dyes unveil interesting behavior of NMPs and descendant progenies. Fluorescence labeled E8 mouse NMPs in the caudal lateral epiblast revealed the preferential contribution of NMPs toward the E9 dorsal neural tube ${ }^{36)}$. We have also demonstrated that genetically labeled E10 mouse NMP cells preferentially contribute toward the dorsal E12 lumbar neural tube ${ }^{44)}$. Thus, these data suggest that either the dorsal and ventral neural tubes have different developmental timings, which may account for the preferential contribution of NMPs, or NMPs consist of distinct sub-populations with distinct differentiation preferences toward neural tube sub-domains. On the other hand, chick NMPs emerge at the Hamburger and Hamilton $(\mathrm{HH}) 8$ posterior epiblast and persist until $\mathrm{HH} 27^{34,39)}$. Between stages HH8 and HH17, NMPs produce both uni-fated mesodermal and neural precursors with selfrenewal and subsequent lineage differentiation. Interestingly, unlike those of mouse, these uni-fated neural precursors have the potential to produce mesodermal cells in addition to neural cells at later stages (HH19 onward $)^{23)}$. This finding in the chick model raises the argument that Sox 2 and Brachyury $\mathrm{T}$ (BraT) are not general markers for NMPs, which foster the need to identify the unique molecular signature for NMPs. In parallel, comparative analysis of the spatiotemporal contribution of NMPs during mouse and chick axial elongation showed differences in localization of Sox $2^{+}$and $\mathrm{BraT}^{+}$cell populations ${ }^{44)}$. While chick Sox $2^{+}$and $\mathrm{BraT}^{+}$cells are restricted to the caudal epiblast, mouse Sox $2^{+}$and $\mathrm{BraT}^{+}$cells are detected in a broad fashion along the elongating neural tube. In zebrafish, NMPs exhibit distinct behavior, and single cell track- ing revealed two NMP populations; the initial population is located near the blastodermal cells close to the marginal zone during gastrulation, whereas the second NMP population in the tail bud is rather quiescent with a low proliferation fate during late somitogenesis ${ }^{3)}$. Unlike the NMPs in mice and chicks, tail-bud NMPs in zebrafish undergo delay in contributing to the neural and mesodermal compartments. A recent mouse study suggests different molecular differentiation and interpretation of the role of NMPs in axial elongation ${ }^{36)}$. Lineage tracing, fluorescent labeling, and conditional knockout experiments suggest that posterior BraT $^{+}$cells are the origin of Sox $2^{+}$and $\mathrm{BraT}^{+}$cell populations that are prone to differentiate toward neural lineage only ${ }^{36)}$. These vast differences among vertebrates raise the challenge of uncovering the precise cellular origin of NMPs toward spinal cord growth across species. Although it is widely accepted that chick, and not mouse axial elongation closely resembles human axial elongation ${ }^{9)}$, it is important to note which model closely mimics human spinal cord development for future disease modeling studies.

Lack of suitable molecular markers for NMPs has added to the ambiguity and complexity of the inter-species comparisons of data. Currently, the co-expression of early neural and mesodermal markers, Sox 2 and BraT is widely used to identify the NMPs in the posterior tissues of human, mouse, and chick embryos as well as in vitro. Recently, however, Nkx1-2 was reported to mark the mouse NMPs throughout the body axis elongation $^{41)}$. While NMPs co-express Sox 2 and BraT markers, the expression level of these molecular markers appears to be associated with their lineage specification (Fig. 2). For instance, early mesodermal restricted progenitors co-express a Sox $2^{\text {low }} /$ $\mathrm{BraT}^{\text {high }} / \mathrm{Nkx1}-2^{\text {low }}$ state, whereas early neural restricted progenitors express a Sox $2^{\text {high }} / \mathrm{BraT}^{\text {low }} / \mathrm{Nkx1} 2^{\text {high }}$ state. Although single-cell RNA-sequence analysis of NMPs and their immediate descendants revealed more complete molecular signatures ${ }^{15,24)}$, the ultimate single molecular marker to locate authentic NMP populations is still missing. Identification of valuable marker sets with new approaches, such as multiplex imaging ${ }^{27,28)}$, may soon be applied in this field to clarify these, and related issues.

\section{SPINAL NEURAL SELECTION AND DIFFEREN- TIATION FROM NMPS}

The identification of NMPs in the developing embryos was 


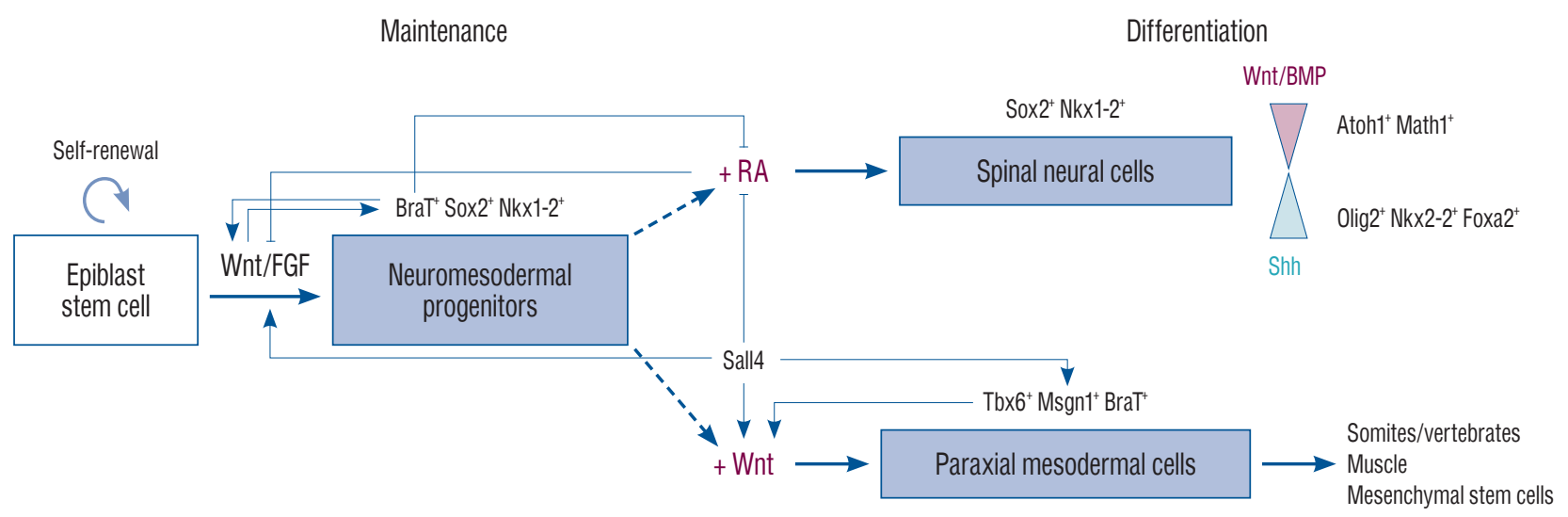

Fig. 2. Differentiation of neuromesodermal progenitors and their molecular niche during the formation of posterior tissues. Overview of the molecular niche demonstrating the relationship between signaling and genes during the differentiation of NMPs toward neural and mesodermal lineages. Both Wnt and FGF signals in the primitive streak promote the induction of NMPs. RA emanating from the newly formed somites inhibits Wnt/FGF signaling that maintains NMP self-renewal and fosters neural cell differentiation from NMPs. Newly formed neural cells in the neural tube respond to shh emanating from the notochord and Wnt/BMP emanating from the roof plate to differentiate toward motor neurons and sensory neurons/neural crest cells, respectively. Wnt activates BraT expression, which in turn promotes Wnt expression, establishing a positive feedback loop in NMPs. BraT itself can inhibit RA signaling. NMPs exposed to high Wnt differentiate toward paraxial mesodermal cells which maintain BraT expression but start to express Tbx6 and Msgn1. Tbx6 itself can activate Wnt signaling, creating a positive feedback loop in paraxial mesodermal cells. These cells later have the capacity to induce somites which become the vertebrates, or to generate muscle and mesenchymal stem cells. Sall4 acts upstream to induce mesodermal cells from NMPs via activation of Wnt and inhibition of RA signaling. Sall4 itself directly interacts with Wnt/FGF to maintain the NMP population. FGF : fibroblast growth factor, BraT : Brachyury T, RA : retinoic acid, NMPs : neuromesodermal progenitors.

surprisingly unexpected, and many experts in the field performed experiments in multiple model organisms. Many of these experiments, including tissue ablation ${ }^{36)}$, cell transplantation $^{17,49)}$, cell labeling with vital dyes ${ }^{23)}$ and genetic lineage tracing $^{14,44)}$ were conducted to highlight the spatial and temporal contributions of NMPs in vertebrates, especially to the neural posterior body. To generate the neural tissues, NMPs first enter the pre-neural tube state where cells downregulate the expression of BraT while maintaining high Sox 2 expression and Nkx1-2 (Fig. 2). These cells are then patterned with a set of specific transcription factors to define the dorsal and ventral identities in response to patterning signals. For instance, Wnt and bone morphogenetic protein (BMP) signaling promotes the expression of Atohl and Math1 to define dorsal neural cells ${ }^{21,38)}$, concurrent opposing Shh signaling induces distinct sets of transcription factors including Olig2 and later Nkx2.2 and Foxa2 to control the ventral identity of NMP-derived neural progenitor cells ${ }^{10)}$.

Genetic studies have provided insights into the regulatory mechanisms of the maintenance and fate choice of NMPs. Despite the difference in vertebrate spinal axial elongation, the molecular mechanisms required for posterior extension seem to be conserved to some degree ${ }^{33)}$. At the molecular level, Wnt/ $\beta$-catenin signaling maintains the proliferation state of $\mathrm{NMPs}^{8}$. Furthermore, $\mathrm{Cdx}$ genes and fibroblast growth factor (FGF) signaling are shown to interact with $\mathrm{Wnt} / \beta$-catenin signaling to maintain NMPs for promoting body elongation ${ }^{2,11}$. Interestingly, all in vitro protocols for inducing NMPs from embryonic stem cells (ESCs) involve exposure to a Wnt agonist over certain time periods. NMP-directed differentiation into the mesodermal fate is dependent on Wnt/ $/$-catenin signaling, and interaction with BraT to induce Tbx6 expression in progenitor state ${ }^{22}$. Recently, Sall4 (gene) dependent Wnt/ $\beta$-catenin signaling was shown to promote mesodermal differentiation at the expense of neural differentiation ${ }^{48}$. The detailed molecular pathways to regulate the fate of NMPs are discussed below. In summary, Wnt signaling not only maintains the NMP state but also promotes the mesodermal fate upon interaction with the above genes. Retinoic acid (RA) signaling is another signaling pathway controlled by NMPs that is required for the body axis extension (Fig. 2). RA is secreted from somites to balance the differentiation of NMPs and control the axial elongation. Genetic studies in mice showed that embryos with mutant Aldh1a2 have significant induction of mesodermal lineages at the expense of spinal cord lineages ${ }^{8)}$. These embryos exhibit ectopic expression of $F g f 8$ extending to 
the developing trunk tissues, suggesting that RA acts as an antagonist to FGF signaling which is required for NMP expansion and proper axial elongation.

NMP differentiation toward neural fate is RA signaling dependent $^{7)}$, where RA inhibits Wnt/ $\beta$-catenin signaling during neural tube elongation to restrict the mesodermal fate of $\mathrm{NMPs}^{45)}$ (Fig. 2). Sall4 encodes zinc finger transcription factors and is strongly expressed in the caudal part of the body. A recent genetic study showed that a mutant conditional Sall4 mouse knockout exhibited enlarged posterior neural tissue with significant depletion in NMPs ${ }^{48}$. Sall4 mutant embryos display expanded expression of Nkx1-2, which is explicitly expressed in E8 pre-neural tube cells as well as a significant increase in motor neuron induction. It is essential to identify the molecular pathways that promote the rapid induction of motor neurons from human NMPs. This will facilitate preclinical studies such as drug screenings for spinal motor neuron degeneration. The generation and characterization of spinal neural cells via NMPs is an upcoming topic of interest for potential therapeutic applications. This was approached, but with limited outcome, when researchers illustrated the transcriptomic profile of neural selection and differentiation from human ESC-NMPs in vitro ${ }^{511}$. Although it is normal to have increased expression of RA signaling related genes due to exogenous RA activation, the spontaneous induction of BMP and Shh pathway genes among neural cells was interesting. This suggests the potential of these cells to exogenously respond to exogenous BMP and Shh treatments to generate sensory and motor neurons, respectively. It also suggests the ability of neural cells with NMP origin to self-pattern, which can be useful for generating mini spinal cord tissue using organoid technology ${ }^{25)}$.

\section{SEGMENTATION AND MESODERM SPECIFICA- TION WITH NEUROMESODERMAL ORIGIN}

The progressive elongation of posterior tissues is best visualized by observing somitogenesis, the process of somite pair formation from paraxial mesodermal segment differentia$\operatorname{tion}^{20)}$. During the transition of NMPs into the mesodermal fate, they first migrate rostrally and downregulate Sox 2 expression to differentiate into paraxial mesodermal cells that express BraT and Tbx6. Rostrally, these cells, under the con- trol of Wnt that promotes further differentiation, in which Hes7 coordinates oscillatory gene expression patterns to sequentially form paired somites ${ }^{20)}$. The continuous supply of paraxial mesodermal cells from NMPs sustains the formation of somites and ensures the development of a proper length of the posterior tissues.

Although BraT is expressed in pre-somitic mesodermal cells only, a complete loss of BraT results in short tail, severely truncated embryos, and often, lethality ${ }^{18,32}$. Tailless phenotype can be seen across species, including mouse, zebrafish, and dogs. There is accumulating evidence that BraT establishes a niche of signaling environment to facilitate mesodermal specification from cells lacking key mesodermal genes, and subsequently, proper somitogenesis $^{30}$. In zebrafish, mice, and humans, BraT directly activates the transcription of Wnt ligands to sustain $W n t / \beta$-catenin signaling activation, which in turn has a positive autoregulatory loop for BraT expression ${ }^{12,35)}$ (Fig. 2). In addition, BraT acts as an upstream inhibitor of the $c y$ p26a1 enzyme to degrade RA that inhibits mesodermal cell specification from $\mathrm{NMPs}^{311}$. Together, this molecular environment promotes the differentiation of NMPs into paraxial mesodermal cells (Fig. 2). Defects in this niche across all vertebrates result in somite loss accompanied by a significant induction of Sox $2^{+}$neural progenitor cells. The process of mesodermal specification usually occurs during gastrulation and later in the posterior tail bud, where BraT and Wnt continue to express and function to induce new paraxial mesodermal progenitors from NMPs. This embryonic process was later recapitulated in vitro using NMP cell culture ${ }^{16,17)}$. In summary, the interaction between BraT and Wnt to repress RA signaling is essential for inducing new mesodermal segments from NMPs, which are critical for segmentation, somitogenesis, and proper axial elongation of the posterior tissues.

Gene expression profile methods such as RNA-sequencing and microarrays are applied to uncover the molecular mechanism of mesodermal specification from $\mathrm{NMPs}^{51)}$. At the gene level, multiple genes were subsequently identified to be expressed in paraxial mesodermal cells and not in NMPs, including Tbx6 ${ }^{42)}$, Msgn1 ${ }^{13)}$, and Sall4 ${ }^{48)}$. Tbx6 regulates NMP fate decisions for its differentiation into the mesodermal fate. Tbx6 and Wnt signaling synergistically interact to induce paraxial mesodermal cells from the NMPs in the tail bud ${ }^{19)}$. The Tbx6 function restricts the neurogenic NMP pool to the chordoneural hinge region, as evidenced in the conditional knock- 
out mouse of Tbx6 from NMPs, which resulted in an increased number of ectopic neural tubes during the transition of trunk to tail development ${ }^{22)}$. On the other hand, the transcription factor Sall4 acts upstream to maintain Wnt-FGF interaction for the self-renewal regulatory system of NMPs (Fig. 2). Similar to Tbx6, Sall4 conditional knockout from NMPs resulted in reduced paraxial mesodermal cells, and perturbed the molecular niche for sustaining paraxial mesodermal cell production and subsequent axial elongation arrest ${ }^{48)}$. Transcriptomic analysis revealed that Sall4 knockout affected several genes, including Msgn1, Hes7, and Wnt5a, all of which are required for nascent mesoderm differentiation ${ }^{5)}$. Msgn1 functions downstream of Wnt signaling and is necessary and sufficient for paraxial mesodermal differentiation from NMPs ${ }^{5}$. It activates key mesodermal progenitor markers, including Tbx6, Pdgfra, and Gata4. Forced expression of Msgn1 has been shown to substantially reduce the contribution of NMPs toward neural progeny ${ }^{5}$. The reduced number of neural cells is accompanied by a correspondingly dramatic increase in paraxial mesodermal cells ${ }^{5)}$. An in vitro study demonstrated that NMPs can be induced in Msgn1 knockout ESCs but cannot efficiently differentiate into paraxial mesodermal cells ${ }^{15}$. Interestingly, a knockout of Sall4 significantly downregulated Msgn1 expression in the posterior tissue ${ }^{48)}$. To summarize the mesodermal molecular network (Fig. 2), Sall4 is upstream of Wnt/ $\beta$-catenin signaling, which itself acts upstream to Tbx6 and Msgn1. In other words, Sall4 directly regulates Tbx6 and Msgn1 expression through Wnt/ $\beta$-catenin signaling. Although the Sall4 knockout showed a defect in Wnt/FGF interaction that is essential to maintain NMPs, Sall4 CHIP-Seq experiment did not show a direct interaction with BraT and Sox2. Hence, it is unlikely that a balanced expression of Sall4 is essential to maintain the self-renewal properties of NMPs; its upstream expression may be needed to trigger the switch of NMP fate toward the mesoderm. Therefore, network analysis is a preferable option for decoding the regulatory basis of axial NMP self-renewal and identifying a unique marker.

\section{CONCLUSION AND PERSPECTIVES}

NMPs have come a long way from their original description as "axial stem cells", to their central importance for developing the CNS. They are now considered the authentic cell pop- ulation for generating the caudal-CNS, the spinal cord. While we have a good understanding of the role of NMPs in development, knowledge of their definitive marker, their exact level of contribution in the CNS, and of which domain in the CNS they interact with the neuroectodermal cell lineage, is still unknown. It is also unclear whether other cell types contribute to the NMP population in the caudal tail bud, or if they exhibit such a high capacity of self-renewal to meet the demand of axial elongation throughout development.

The discovery of NMPs has changed the fundamental belief that the three-germ layer formation terminates strictly during early development and ectoderm/mesoderm lineage segregation. In fact, in this stem cell engineering era, trans-differentiation and de-differentiation are experimentally inducible, and it is no wonder that developmentally multi-potent cells can be reprogrammed. As we have described here, there are two lineage routes for making the CNS, where neuroectodermal cells generate the rostral neural plate, but caudal neural cells are developmentally produced from NMPs. The recapitulation of the caudal developmental process appears to favor the generation of caudal neural structures such as the spinal cord. Likewise, recent studies investigating the development of human trunk parts have highlighted the importance of NMP induction in vitro, which led to the high-yield production of multiple spinal cell types, including spinal motoneurons ${ }^{17,25,29)}$. This allows us to understand human caudal development based on human organoids ${ }^{25)}$. Considering the fact that there are many developmental and pathological conditions requiring detailed disease modeling and regenerative therapy in the spinal cord, fundamental information about NMPs will be of great value in the diagnosis and therapy of these related clinical conditions.

\section{CONFLICTS OF INTEREST}

No potential conflict of interest relevant to this article was reported.

\section{INFORMED CONSENT}

This type of study does not require informed consent. 


\section{AUTHOR CONTRIBUTIONS}

\author{
Conceptualization : MRS, WS \\ Visualization : MRS, JHL \\ Writing - original draft : MRS, JHL, WS \\ Writing - review \& editing: WS
}

\section{ORCID}

Mohammed R. Shaker https://orcid.org/0000-0002-3675-4598

Ju-Hyun Lee https://orcid.org/0000-0002-1006-7482

Woong Sun https://orcid.org/0000-0003-1792-4894

\section{- Acknowledgements}

MRS is supported by the MRFF Leukodystrophy Flagship Massimo's Mission (EPCD000034). JHL and WS are supported by the Brain Research Program through the National Research Foundation (NRF), which was funded by the Korean Ministry of Science, ICT \& Future Planning (NRF2015M3C7A1028790, NRF-2017M3A9B3061308, and NRF2017M3C7A1047654).

\section{References}

1. Aires $R$, Dias $A$, Mallo $M$ : Deconstructing the molecular mechanisms shaping the vertebrate body plan. Curr Opin Cell Biol 55 : 81-86, 2018

2. Amin S, Neijts R, Simmini S, van Rooijen C, Tan SC, Kester L, et al. : Cdx and $\mathrm{T}$ brachyury co-activate growth signaling in the embryonic axial progenitor niche. Cell Rep 17 : 3165-3177, 2016

3. Attardi A, Fulton T, Florescu M, Shah G, Muresan L, Lenz MO, et al. : Neuromesodermal progenitors are a conserved source of spinal cord with divergent growth dynamics. Development 145 : dev166728, 2018

4. Berenguer M, Lancman JJ, Cunningham TJ, Dong PDS, Duester G : Mouse but not zebrafish requires retinoic acid for control of neuromesodermal progenitors and body axis extension. Dev Biol 441 : 127-131, 2018

5. Chalamalasetty RB, Garriock RJ, Dunty WC Jr, Kennedy MW, Jailwala $\mathrm{P}, \mathrm{Si}$, et al. : Mesogenin 1 is a master regulator of paraxial presomitic mesoderm differentiation. Development 141 : 4285-4297, 2014

6. Costanzo R, Watterson RL, Schoenwolf GC : Evidence that secondary neurulation occurs autonomously in the chick embryo. J Exp Zool 219 : 233-240, 1982
7. Cunningham TJ, Colas A, Duester $\mathrm{G}$ : Early molecular events during retinoic acid induced differentiation of neuromesodermal progenitors. Biol Open 5 : 1821-1833, 2016

8. Cunningham TJ, Kumar S, Yamaguchi TP, Duester G : Wnt8a and Wnt3a cooperate in the axial stem cell niche to promote mammalian body axis extension. Dev Dyn 244 : 797-807, 2015

9. Dady A, Havis E, Escriou V, Catala M, Duband JL : Junctional neurulation: a unique developmental program shaping a discrete region of the spinal cord highly susceptible to neural tube defects. J Neurosci 34 : 13208-13221, 2014

10. Dessaud E, McMahon AP, Briscoe J : Pattern formation in the vertebrate neural tube: a sonic hedgehog morphogen-regulated transcriptional network. Development 135 : 2489-2503, 2008

11. Diez del Corral R, Morales AV : The multiple roles of FGF signaling in the developing spinal cord. Front Cell Dev Biol 5 : 58, 2017

12. Evans AL, Faial T, Gilchrist MJ, Down T, Vallier L, Pedersen RA, et al. : Genomic targets of Brachyury $(\mathrm{T})$ in differentiating mouse embryonic stem cells. PLoS One 7 : e33346, 2012

13. Fior R, Maxwell AA, Ma TP, Vezzaro A, Moens CB, Amacher SL, et al. : The differentiation and movement of presomitic mesoderm progenitor cells are controlled by Mesogenin 1. Development 139 : 4656-4665, 2012

14. Garriock RJ, Chalamalasetty RB, Kennedy MW, Canizales LC, Lewandoski M, Yamaguchi TP : Lineage tracing of neuromesodermal progenitors reveals novel Wnt-dependent roles in trunk progenitor cell maintenance and differentiation. Development 142 : 1628-1638, 2015

15. Gouti M, Delile J, Stamataki D, Wymeersch FJ, Huang Y, Kleinjung J, et al. : A gene regulatory network balances neural and mesoderm specification during vertebrate trunk development. Dev Cell 41 : 243-261.e7, 2017

16. Gouti M, Metzis V, Briscoe J : The route to spinal cord cell types: a tale of signals and switches. Trends Genet 31 : 282-289, 2015

17. Gouti M, Tsakiridis A, Wymeersch FJ, Huang Y, Kleinjung J, Wilson V, et al. : In vitro generation of neuromesodermal progenitors reveals distinct roles for Wnt signalling in the specification of spinal cord and paraxial mesoderm identity. PLoS Biol 12 : e1001937, 2014

18. Halpern ME, Ho RK, Walker C, Kimmel CB : Induction of muscle pioneers and floor plate is distinguished by the zebrafish no tail mutation. Cell 75 : 99-111, 1993

19. Hofmann M, Schuster-Gossler K, Watabe-Rudolph M, Aulehla A, Herrmann BG, Gossler A : Wnt signaling, in synergy with T/TBX6, controls Notch signaling by regulating DII1 expression in the presomitic mesoderm of mouse embryos. Genes Dev 18 : 2712-2717, 2004

20. Hubaud $A$, Pourquié 0 : Signalling dynamics in vertebrate segmentation. Nat Rev Mol Cell Biol 15 : 709-721, 2014

21. Ille F, Atanasoski $S$, Falk $S$, Ittner LM, Märki D, Büchmann-Møller $S$, et al. : Wnt/BMP signal integration regulates the balance between proliferation and differentiation of neuroepithelial cells in the dorsal spinal cord. Dev Biol 304 : 394-408, 2007

22. Javali A, Misra A, Leonavicius K, Acharyya D, Vyas B, Sambasivan R : Co-expression of Tbx6 and Sox 2 identifies a novel transient neuromeso- 
derm progenitor cell state. Development 144 : 4522-4529, 2017

23. Kawachi T, Shimokita E, Kudo R, Tadokoro R, Takahashi $Y$ : Neural-fated self-renewing cells regulated by Sox 2 during secondary neurulation in chicken tail bud. Dev Biol 461 : 160-171, 2020

24. Koch F, Scholze M, Wittler L, Schifferl D, Sudheer S, Grote P, et al. : Antagonistic activities of Sox 2 and brachyury control the fate choice of neuro-mesodermal progenitors. Dev Cell 42 : 514-526.e7, 2017

25. Lee JH, Shin H, Shaker MR, Kim HJ, Kim JH, Lee N, et al. : Human spinal cord organoids exhibiting neural tube morphogenesis for a quantifiable drug screening system of neural tube defects. bioRxiv, 2020 [Epub ahead of print]

26. Lee JY, Lee ES, Kim SP, Lee MS, Phi JH, Kim SK, et al. : Neurosphere formation potential resides not in the caudal cell mass, but in the secondary neural tube. Int J Dev Biol 61 : 545-550, 2017

27. Li W, Germain RN, Gerner MY : Multiplex, quantitative cellular analysis in large tissue volumes with clearing-enhanced 3D microscopy (Ce3D). Proc Natl Acad Sci U S A 114 : E7321-E7330, 2017

28. Lin JR, Izar B, Wang S, Yapp C, Mei S, Shah PM, et al. : Highly multiplexed immunofluorescence imaging of human tissues and tumors using t-CyCIF and conventional optical microscopes. Elife 7 : e31657, 2018

29. Lippmann ES, Williams CE, Ruhl DA, Estevez-Silva MC, Chapman ER, Coon JJ, et al. : Deterministic HOX patterning in human pluripotent stem cell-derived neuroectoderm. Stem Cell Reports 4 : 632-644, 2015

30. Martin BL : Factors that coordinate mesoderm specification from neuromesodermal progenitors with segmentation during vertebrate axial extension. Semin Cell Dev Biol 49 : 59-67, 2016

31. Martin BL, Kimelman D : Brachyury establishes the embryonic mesodermal progenitor niche. Genes Dev 24 : 2778-2783, 2010

32. Martin BL, Kimelman D : Regulation of canonical Wnt signaling by Brachyury is essential for posterior mesoderm formation. Dev Cell 15 : 121-133, 2008

33. Martin $\mathrm{BL}$, Kimelman $\mathrm{D}$ : Wht signaling and the evolution of embryonic posterior development. Curr Biol 19 : R215-R219, 2009

34. McGrew MJ, Sherman A, Lillico SG, Ellard FM, Radcliffe PA, Gilhooley $\mathrm{HJ}$, et al. : Localised axial progenitor cell populations in the avian tail bud are not committed to a posterior Hox identity. Development 135 : 2289-2299, 2008

35. Morley RH, Lachani K, Keefe D, Gilchrist MJ, Flicek P, Smith JC, et al. : A gene regulatory network directed by zebrafish No tail accounts for its roles in mesoderm formation. Proc Natl Acad Sci U S A 106 : 38293834, 2009

36. Mugele D, Moulding DA, Savery D, Molè MA, Greene ND, MartinezBarbera JP, et al. : Genetic approaches in mice demonstrate that neuromesodermal progenitors express T/Brachyury but not Sox2. bioRxiv, 2018 [Epub ahead of print]

37. Müller F, O'rahilly $R$ : The primitive streak, the caudal eminence and related structures in staged human embryos. Cells Tissues Organs 177 : 2-20, 2004

38. Mulvaney J, Dabdoub A : Atoh1, an essential transcription factor in neurogenesis and intestinal and inner ear development: function, regu- lation, and context dependency. J Assoc Res Otolaryngol 13 : 281293, 2012

39. Olivera-Martinez I, Harada H, Halley PA, Storey KG : Loss of FGFdependent mesoderm identity and rise of endogenous retinoid signalling determine cessation of body axis elongation. PLoS Biol 10 : e1001415, 2012

40. Pai YJ, Abdullah NL, Mohd-Zin SW, Mohammed RS, Rolo A, Greene ND, et al. : Epithelial fusion during neural tube morphogenesis. Birth Defects Res A Clin Mol Teratol 94 : 817-823, 2012

41. Rodrigo Albors A, Halley PA, Storey KG : Lineage tracing of axial progenitors using Nkx1-2CreERT2 mice defines their trunk and tail contributions. Development 145 : dev164319, 2018

42. Sadahiro T, Isomi M, Muraoka N, Kojima H, Haginiwa S, Kurotsu S, et al. : Tbx6 induces nascent mesoderm from pluripotent stem cells and temporally controls cardiac versus somite lineage diversification. Cell Stem Cell 23 : 382-395.e5, 2018

43. Shaker MR, Kim JY, Kim H, Sun W : Identification and characterization of secondary neural tube-derived embryonic neural stem cells in vitro. Stem Cells Dev 24 : 1171-1181, 2015

44. Shaker MR, Lee JH, Kim KH, Kim VJ, Kim JY, Lee JY, et al. : Spatiotemporal contribution of neuromesodermal progenitor-derived neural cells in the elongation of developing mouse spinal cord. bioRxiv, 2020 [Epub ahead of print]

45. Shaker MR, Lee JH, Park SH, Kim JY, Son GH, Son JW, et al. : Anteroposterior Wnt-RA gradient defines adhesion and migration properties of neural progenitors in developing spinal cord. Stem Cell Reports 15 : 898-911, 2020

46. Shimokita E, Takahashi Y : Secondary neurulation: fate-mapping and gene manipulation of the neural tube in tail bud. Dev Growth Differ $53: 401-410,2011$

47. Shum AS, Tang LS, Copp AJ, Roelink H : Lack of motor neuron differentiation is an intrinsic property of the mouse secondary neural tube. Dev Dyn 239 : 3192-3203, 2010

48. Tahara N, Kawakami H, Chen KQ, Anderson A, Yamashita Peterson M, Gong $W$, et al. : Sall4 regulates neuromesodermal progenitors and their descendants during body elongation in mouse embryos. Development 146 : dev177659, 2019

49. Tsakiridis A, Huang Y, Blin G, Skylaki S, Wymeersch F, Osorno R, et al. : Distinct Wnt-driven primitive streak-like populations reflect in vivo lineage precursors. Development 141 : 1209-1221, 2014

50. Tzouanacou E, Wegener A, Wymeersch FJ, Wilson V, Nicolas JF : Redefining the progression of lineage segregations during mammalian embryogenesis by clonal analysis. Dev Cell 17 : 365-376, 2009

51. Verrier L, Davidson L, Gierliński M, Dady A, Storey KG : Neural differentiation, selection and transcriptomic profiling of human neuromesodermal progenitor-like cells in vitro. Development 145 : dev166215, 2018

52. Yang HJ, Lee DH, Lee YJ, Chi JG, Lee JY, Phi JH, et al. : Secondary neurulation of human embryos: morphological changes and the expression of neuronal antigens. Childs Nerv Syst 30 : 73-82, 2014 